\title{
Poster presentations
}

\section{H1-NMR-based metabolomics provides new insights into canine diabetes mellitus}

\section{Claudia Ottka ${ }^{1}$, Elisabeth Müller ${ }^{2}$, Corinna Weber ${ }^{2}$, Hannes Lohi ${ }^{1,3,4}$}

PetBIOMICS Ltd, Helsinki, Finland

LABOKLIN Ltd, Bad Kissingen, Germany

Department of Veterinary Biosciences and Department of

Medical and Clinical Genetics, University of Helsinki, Helsinki,

Finland

4 Folkhälsan Research Center, Helsinki, Finland

\section{OBJECTIVES}

To determine the metabolic changes occurring in canine diabetes mellitus.

\section{METHODS}

We analysed a group of 80 canine serum/heparin plasma samples with markedly elevated fructosamine concentrations and a control group of 25 canine samples with normal clinical chemistry by a canine-specific H1-NMR based metabolomics testing platform. The test and control group results means and medians were calculated, the significance was analysed by Bonferroni-corrected t-test statistics and the changes were compared to the reference ranges of the analysis.

\section{RESULTS}

A multitude of analytes showed significant $(p<0,05)$ differences between the two groups, and abnormal levels compared to analysis reference ranges. These analytes included e.g. isoleucine, leucine, valine, glutamine, phenylalanine, total cholesterol, free cholesterol, LDL cholesterol, VLDL cholesterol, total fatty acids, triglycerides, HDL triglycerides, VLDL triglycerides, glucose, lactate and glycoprotein acetyls.

\section{STATEMENT (CONCLUSIONS)}

This study highlights the wide impact canine diabetes mellitus has on metabolism. These results suggest, that canine diabetes mellitus should not be clinically viewed solely as a disorder of glucose metabolism, but the systemic nature, including an inflammatory component, and metabolic consequences of this disease, such as muscle wasting, should be considered in disease diagnostics and management. The novel comprehensive NMR-metabolomics platform may be more potent in monitoring the disease than any single established parameter. More studies are needed to confirm this hypothesis. 